\title{
FINITE CASIMIR ENERGIES IN RENORMALIZABLE QUANTUM FIELD THEORY*
}

\author{
KIMBALL A. MILTON \\ Department of Physics and Astronomy, \\ University of Oklahoma, \\ Norman, OK 73019-2061 USA \\ E-mail:milton@nhn.ou.edu
}

\begin{abstract}
Quantum vacuum energy has been known to have observable consequences since 1948 when Casimir calculated the force of attraction between parallel uncharged plates, a phenomenon confirmed experimentally with ever increasing precision. Casimir himself suggested that a similar attractive self-stress existed for a conducting spherical shell, but Boyer obtained a repulsive stress. Other geometries and higher dimensions have been considered over the years. Local effects, and divergences associated with surfaces and edges have been investigated by several authors. Quite recently, Graham et al. have re-examined such calculations, using conventional techniques of perturbative quantum field theory to remove divergences, and have suggested that previous self-stress results may be suspect. Here we show that most of the examples considered in their work are misleading; in particular, it is well-known that in two dimensions a circular boundary has a divergence in the Casimir energy for massless fields, while for general dimension $D$ not equal to an even integer the corresponding Casimir energy arising from massless fields interior and exterior to a hyperspherical shell is finite. It has also long been recognized that the Casimir energy for massive fields is divergent for curved boundaries. These conclusions are reinforced by a calculation of the relevant leading Feynman diagram in $D$ dimensions. Divergences do occur in third order, as has been recognized for many years, but this logarithmic divergence is of questionable relevance to real shells.
\end{abstract}

\section{Introduction}

The Casimir effect remains one of the least intuitive consequences of quantum field theory, and stands rather outside the usual development of renormalization theory. This is because it is inherently nonperturbative, in that macroscopic boundary conditions or backgrounds cannot be easily mimicked by perturbative interactions. Its origins go back to the very beginnings of quantum mechanics, because it can be thought of as the change in the zero-point energy when the background is introduced.

After examining the van der Waals interaction between two molecules and between a molecule and a conducting plate ${ }^{1}$ Casimir was challenged by Bohr to interpret this interaction in terms of zero-point energy, 2 and then to recognize that

*Invited talk given at Marcel Grossmann X, Rio de Janeiro, Brazil, July 20-26, 2003 
the zero-point fluctuations of the electromagnetic field implied a force between two such plates. ${ }^{3}$ The attractive nature of this force was obviously consistent with the action-at-a-distance interpretation of it as due to the attraction between fluctuating dipoles making up the material of the plates. But intuition flew out the window when Boyer discovered that the energy, and hence the self-stress, on a perfectly conducting spherical shell of zero thickness was positive or repulsive ${ }^{4}$ Later, it was found that a cylinder was intermediate, giving rise to a small but attractive force $[\overline{5}$

Dimensional dependence was also dramatic. Sen examined a circular boundary in two dimensions and found that the energy was infinite $[6]$ This was later found to be part of a pattern: For a hyperspherical shell in $D$ spatial dimensions, the Casimir energy of a massless scalar field subject to Dirichlet boundary conditions was finite except when $D$ was a positive even integer, where the energy or stress exhibits a simple pole, as seen in Fig. [1] (For $D \leq 0$, branch points occur at the integers.) An intuitive explanation of this, and of the corresponding sign changes, is still lacking.

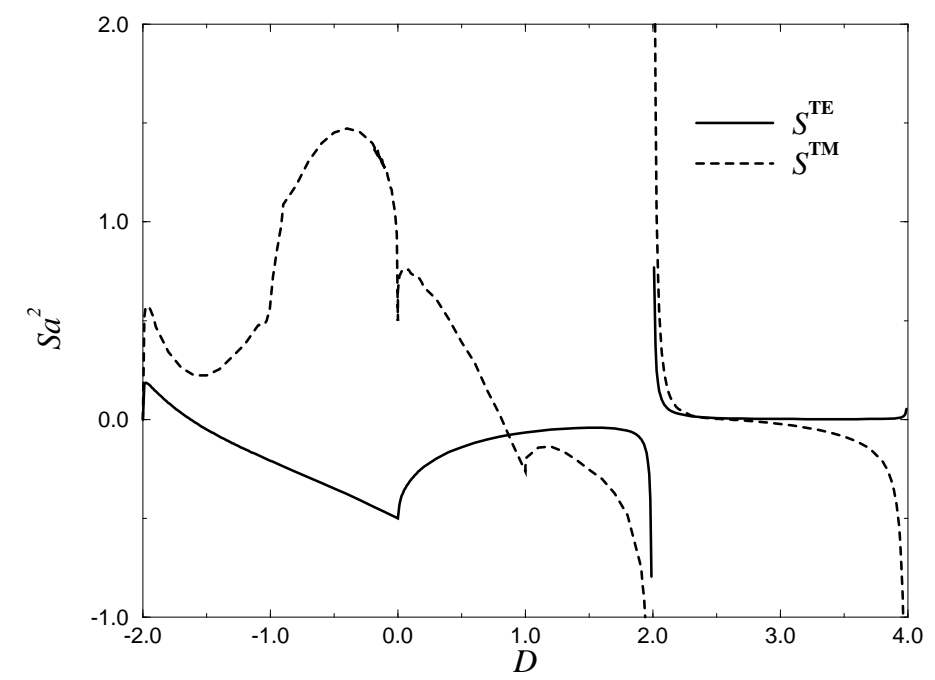

Figure 1. A plot of the TM and TE Casimir stress for $-2<D<4$ on a perfectly conducting spherical shell. For $D<2(D<0)$ the stress $\mathcal{S}^{\mathrm{TM}}\left(\mathcal{S}^{\mathrm{TE}}\right)$ is complex and we have plotted the real part. The TM calculation was given in Ref. 8

Deutsch and Candelas were the first to examine the local effects of fluctuating fields, 9 for other than the geometry of parallel planes, which was considered by Brown and Maclay a decade earlier 10 Typically, surface divergences occur near boundaries, although for flat boundaries with conformally-coupled fields, those di- 
vergences disappear. The reason the global Casimir energy of a (hyper)sphere is finite is that there is a perfect cancellation between the interior and exterior divergences. This perfect cancellation is spoiled if the shell has finite thickness, or if the speed of light is different on the two sides of the boundary ${ }^{11}$ Giving the fluctuating field a mass also yields an unremovable divergence $\frac{12}{2}$ except for the case of plane boundaries.

Recently, Graham et al 13 have questioned these findings. They have developed an approach in which idealized boundary conditions are replaced with interactions with an external (nondynamical) field. Potentially divergent terms are subtracted and replaced by perturbatively calculable Feynman diagrams. After renormalization of these diagrams, the limiting case when the external field becomes a delta function is taken. In this way the results for $D=1$ are reproduced; but the authors find those finite results rather unsatisfactory, so they discuss how their limiting procedure gives rise to a different energy, corresponding, however, to the conventional force.

Then they turn to $D=2$ and find that it is divergent; the implication is that this is a general feature, so that all calculations of Casimir self-stress are called into question. However, as we remarked above, $D=2$ is a singular point. What is called for is a calculation for general $D$. That is the purpose of this talk. For simplicity, our attention will be restricted to scalar fields. We will first, in Sec. 2 re-examine the $D=1$ calculation, and show that the force is completely finite, while the energy density, or more generally, the stress tensor, has a constant divergent part which would be present if the boundaries were not present, and is therefore quite without observable consequence. For parallel plates in $D$ dimensions, unphysical surface divergences appear in the stress tensor (unphysical because they do not contribute to the stress on the plates), which, for zero mass, vanish if the conformal stress tensor is used. Then, in Sec. 3 we re-examine the self-stress on a sphere in three dimensions, using time-splitting to regulate the divergences. The result is, once again, unambiguously finite. The critical calculation is given in Sec. 4 where we review and simplify the diagrammatic subtraction method, and explicitly compute the graph in which two external fields are inserted, in $D$ spatial dimensions. As expected, the result is divergent at $D=2,4,6, \ldots$, but is otherwise finite for $D>$ 3/2. (A divergence, however, will occur in the graph with three insertions $15 / 16$ ) Concluding remarks are offered in Sec. [5] This talk is based largely on Ref. 17

\section{Casimir Effect for Dirichlet Plates}

\subsection{Massless Scalar in $1+1$ Dimensions}

We begin by reconsidering the Casimir effect for a massive scalar field which vanishes on two parallel plates (Dirichlet boundary conditions). Although these considerations are familiar, we will concentrate on the local effect in $1+1$ dimensions in order to make the divergence structure manifest and make contact with the work of Graham et al! 13 
For a massless scalar field $\phi$, the stress tensor is

$$
T^{\mu \nu}=\partial^{\mu} \phi \partial^{\nu} \phi-\frac{1}{2} g^{\mu \nu} \partial_{\lambda} \phi \partial^{\lambda} \phi .
$$

It will be noted that for one spatial dimension, this canonical tensor coincides with the conformal one,

$$
T_{\mu}^{\mu}=0
$$

The scalar field satisfies the free equation

$$
-\partial^{2} \phi=0,
$$

but is subject to the Dirichlet boundary conditions on the plates at $x=0$ and $x=a$ :

$$
\phi(x=0)=\phi(x=a)=0 .
$$

The corresponding Green's function satisfies

$$
-\partial^{2} G\left(x, t ; x^{\prime}, t^{\prime}\right)=\delta\left(x-x^{\prime}\right) \delta\left(t-t^{\prime}\right),
$$

and

$$
G\left(0, t ; x^{\prime}, t^{\prime}\right)=G\left(a, t ; x^{\prime}, t^{\prime}\right)=0 .
$$

Since the Green's function is translationally invariant in time, it is natural to introduce a corresponding Fourier transform,

$$
G\left(x, x^{\prime} ; t-t^{\prime}\right)=\int_{-\infty}^{\infty} \frac{d \omega}{2 \pi} e^{-i \omega\left(t-t^{\prime}\right)} g\left(x, x^{\prime} ; \omega\right)
$$

the reduced Green's function satisfies the ordinary differential equation

$$
-\left(\omega^{2}+\frac{d^{2}}{d x^{2}}\right) g\left(x, x^{\prime} ; \omega\right)=\delta\left(x-x^{\prime}\right) .
$$

We only need the solutions of this equation in two regions:

$$
\begin{aligned}
& g\left(x, x^{\prime} ; \omega\right)=-\frac{\sin \omega x_{<} \sin \omega\left(x_{>}-a\right)}{\omega \sin \omega a}, \quad 0 \leq x, x^{\prime} \leq a, \\
& g\left(x, x^{\prime} ; \omega\right)=\frac{1}{\omega} \sin \omega\left(x_{<}-a\right) e^{i|\omega|\left(x_{>}-a\right)}, \quad a \leq x, x^{\prime} .
\end{aligned}
$$

Here $x_{>}\left(x_{<}\right)$is the greater (lesser) of $x$ and $x^{\prime}$. These are to be compared to the free Green's function, when no plates are present:

$$
g_{0}\left(x, x^{\prime} ; \omega\right)=\frac{i}{2|\omega|} e^{i|\omega|\left|x-x^{\prime}\right|} .
$$

When we recognize that the Green's function is the time-ordered product of the fields,

$$
\left\langle\phi(x, t) \phi\left(x^{\prime}, t^{\prime}\right)\right\rangle=\frac{1}{i} G\left(x, t ; x^{\prime}, t^{\prime}\right),
$$


we see that the vacuum expectation value of the stress tensor may be obtained by applying a differential operator to the Green's function, and then taking the spacetime points to be coincident. For the 00 component, that is, the energy, that differential operator is

$$
\partial_{0} \partial_{0}^{\prime}+\frac{1}{2} \partial^{\lambda} \partial_{\lambda}^{\prime}=\frac{1}{2} \partial_{0} \partial_{0}^{\prime}+\frac{1}{2} \partial_{x} \partial_{x}^{\prime}
$$

and so we obtain between the plates

$$
\begin{aligned}
\left\langle T^{00}\right\rangle & =\left.\int \frac{d \omega}{2 \pi} \frac{1}{2 i}\left(\omega^{2}+\partial_{x} \partial_{x}^{\prime}\right) g\left(x, x^{\prime} ; \omega\right)\right|_{x=x^{\prime}} \\
& =\int \frac{d \omega}{2 \pi} \frac{\omega^{2}}{2} \frac{i}{\omega \sin \omega a}[\sin \omega x \sin \omega(x-a)+\cos \omega x \cos \omega(x-a)] \\
& =\int \frac{d \omega}{2 \pi} \frac{i \omega}{2} \cot \omega a \rightarrow-\frac{1}{4 \pi} \int_{-\infty}^{\infty} d \zeta \zeta \operatorname{coth} \zeta a .
\end{aligned}
$$

Here, in the last step we have made the complex frequency rotation,

$$
\omega \rightarrow i \zeta
$$

We notice that this last integral in Eq. (14) does not exist. This is because for large $\zeta$ the hyperbolic cotangent approaches unity. If we subtract off this limiting value we obtain a finite result:

$$
\left\langle T^{00}\right\rangle \rightarrow-\frac{1}{2 \pi} \int_{0}^{\infty} d \zeta \zeta(\operatorname{coth} \zeta a-1)=-\frac{1}{\pi} \int_{0}^{\infty} \zeta d \zeta \frac{1}{e^{2 \zeta a}-1}=-\frac{\pi}{24 a^{2}} .
$$

The energy is obtained from this by multiplying by the distance between the plates:

$$
E=-\frac{\pi}{24 a}
$$

which is the well-known Lüscher potential 18

In the same way we can calculate the vacuum expectation value of the $x x$ component of the stress. The relevant differential operator

$$
\partial_{x} \partial_{x}^{\prime}-\frac{1}{2} \partial_{\lambda} \partial^{\prime \lambda} \rightarrow \frac{1}{2}\left(\omega^{2}+\partial_{x} \partial_{x}^{\prime}\right)
$$

is unchanged, so we obtain the same result as for $\left\langle T^{00}\right\rangle$. The off-diagonal terms $\left\langle T^{0 x}\right\rangle$ result from the application of the symmetric differential operator

$$
\frac{1}{2}\left(\partial^{0} \partial^{\prime x}+\partial^{x} \partial^{\prime 0}\right)
$$

so are necessarily zero. Keeping the divergent term we subtracted off, the result for the stress tensor between the plates, $0 \leq x, x^{\prime} \leq a$, is

$$
\left\langle T^{\mu \nu}\right\rangle=\left[u_{\mathrm{vac}}-\frac{\pi}{24 a^{2}}\right]\left(\begin{array}{ll}
1 & 0 \\
0 & 1
\end{array}\right),
$$


where the divergent term is

$$
u_{\mathrm{vac}}=-\frac{1}{2 \pi} \int_{0}^{\infty} d \zeta \zeta
$$

$\left\langle T^{\mu \nu}\right\rangle$ is traceless, as required by conformal symmetry:

$$
\left\langle T_{\mu}^{\mu}\right\rangle=0,
$$

If we follow the same operations to find the stress tensor outside the plates from Eq. (10) we obtain

$$
\begin{aligned}
\left\langle T^{00}\right\rangle & =\left\langle T_{x x}\right\rangle=\frac{1}{4 \pi i} \int \frac{d \omega}{\omega}\left[i \omega|\omega| \cos \omega(x-a) e^{i|\omega|(x-a)}+\omega^{2} \sin \omega(x-a) e^{i|\omega|(x-a)}\right] \\
& =\frac{1}{4 \pi} \int_{-\infty}^{\infty} d \omega|\omega|=-\frac{1}{2 \pi} \int_{0}^{\infty} d \zeta \zeta=u_{\mathrm{vac} .}
\end{aligned}
$$

That is, in the two regions outside the plates, $x<0$ or $x>a$,

$$
\left\langle T^{\mu \nu}\right\rangle=u_{\mathrm{vac}}\left(\begin{array}{ll}
1 & 0 \\
0 & 1
\end{array}\right) .
$$

This is exactly the stress tensor that would be found everywhere if the free Green's function $g_{0}$ in Eq. (11) were used. This means that the force on one of the plates is completely finite and unambiguous, because it is given by the discontinuity of the $x x$ component of the stress tensor across the plate (which follows immediately from the physical meaning of the stress tensor in terms of the flux of momentum):

$$
F=\left.\left\langle T_{x x}\right\rangle\right|_{x=a-}-\left.\left\langle T_{x x}\right\rangle\right|_{x=a+}=-\frac{\pi}{24 a^{2}} .
$$

Since energies are undefined up to a constant, without any loss of generality we may take the stress tensor to be completely finite:

$$
\left\langle T^{\mu \nu}(x)\right\rangle \rightarrow\left\{\begin{array}{c}
-\frac{\pi}{24 a^{2}}\left(\begin{array}{ll}
1 & 0 \\
0 & 1
\end{array}\right), \quad 0 \leq x \leq a, \\
0, \quad x<0 \quad \text { or } \quad x>a .
\end{array}\right.
$$

\subsection{Massless Scalar in 3+1 Dimensions}

In higher dimensions, surface divergences appear. These were discussed in detail in Ref! 19 .

In three space dimensions, the use of the canonical stress tensor (11) leads to the following expression for the vacuum expectation value of the energy density,

$$
\left\langle T^{00}\right\rangle=\int \frac{d \omega}{2 \pi} \frac{d^{2} k}{(2 \pi)^{2}}\left\langle t^{00}\right\rangle
$$

where we have Fourier transformed both in frequency and transverse momentum. If we take the plates to be located at $z=0$ and at $z=a$, we obtain $\left\langle t^{00}\right\rangle$ by applying the differential operator

$$
\frac{1}{2}\left(\partial_{0} \partial_{0}^{\prime}+\partial_{x} \partial_{x}^{\prime}+\partial_{y} \partial_{y}^{\prime}+\partial_{z} \partial_{z}^{\prime}\right) \rightarrow \frac{1}{2}\left(\omega^{2}+k^{2}+\partial_{z} \partial_{z}^{\prime}\right)
$$


to the Green's function (9) with $\omega \rightarrow \lambda \equiv \sqrt{\omega^{2}-k^{2}}$,

$$
0 \leq x, x^{\prime} \leq a: \quad g\left(x, x^{\prime} ; \lambda\right)=-\frac{\sin \lambda x_{<} \sin \lambda\left(x_{>}-a\right)}{\lambda \sin \lambda a} .
$$

The result,

$$
\left\langle t^{00}\right\rangle=-\frac{1}{2 i \lambda \sin \lambda a}\left[\omega^{2} \cos \lambda a-k^{2} \cos \lambda(2 z-a)\right],
$$

is evaluated by making a Euclidean rotation,

$$
\omega \rightarrow i \zeta, \quad \lambda \rightarrow i \kappa,
$$

and introducing polar coordinates in the $\zeta, k$ plane,

$$
\zeta=\kappa \cos \theta, \quad k=\kappa \sin \theta,
$$

so

$$
\begin{aligned}
\left\langle T^{00}\right\rangle(z) & =-\frac{1}{4 \pi^{2}} \int_{0}^{\infty} \kappa d \kappa \int_{0}^{\pi / 2} d \theta \kappa^{2} \frac{\sin \theta}{\sinh \kappa a}\left[\cos ^{2} \theta \cosh \kappa a+\sin ^{2} \theta \cosh \kappa(2 z-a)\right] \\
& =-\frac{1}{12 \pi^{2}} \int_{0}^{\infty} d \kappa \kappa^{3} \frac{1}{\sinh \kappa a}[\cosh \kappa a+2 \cosh \kappa(2 z-a)] \\
& =-\frac{1}{6 \pi^{2}} \int_{0}^{\infty} d \kappa \kappa^{3}\left(\frac{1}{e^{2 \kappa a}-1}+\frac{1}{2}+\frac{e^{2 \kappa z}+e^{2 \kappa(a-z)}}{e^{2 \kappa a}-1}\right) .
\end{aligned}
$$

Notice that the second term in the last integrand here corresponds to a constant energy density, independent of $a$, so as before it may be discarded as irrelevant. If we integrate the third term over $z$,

$$
\int_{0}^{a} d z\left[e^{2 \kappa z}+e^{2 \kappa(a-z)}\right]=\frac{1}{\kappa}\left[e^{2 \kappa a}-1\right],
$$

we obtain another (divergent) constant term, so the only part of the vacuum energy corresponding to an observable force is that coming from the first term:

$$
\int_{0}^{a} d z\left\langle T^{00}\right\rangle(z)=-\frac{a}{6 \pi^{2}} \int_{0}^{\infty} d \kappa \frac{\kappa^{3}}{e^{2 \kappa a}-1}=-\frac{\pi^{2}}{1440 a^{3}},
$$

which is the well-known Casimir energy/area for a massless scalar field subject to Dirichlet boundary conditions, one-half that for an electromagnetic field.

In general, we have

$$
\left\langle T^{00}\right\rangle(z)=u+g(z)
$$

where

$$
u=-\frac{\pi^{2}}{1440 a^{4}}, \quad g(z)=-\frac{1}{6 \pi^{2}} \frac{1}{16 a^{4}} \int_{0}^{\infty} d y y^{3} \frac{e^{y z / a}+e^{y(1-z / a)}}{e^{y}-1} .
$$

If we expand the denominator in a geometric series,

$$
\frac{1}{e^{y}-1}=\frac{e^{-y}}{1-e^{-y}}=\sum_{n=1}^{\infty} e^{-n y}
$$


we can express $g$ in terms of the generalized or Hurwitz zeta function,

$$
\zeta(s, a) \equiv \sum_{n=0}^{\infty} \frac{1}{(n+a)^{s}}, \quad a \neq \text { a negative integer }
$$

as follows:

$$
g(z)=-\frac{1}{16 \pi^{2} a^{4}}[\zeta(4, z / a)+\zeta(4,1-z / a)]
$$

This function is plotted in Fig. 2 where it will be observed that it diverges quartically as $z \rightarrow 0, a$. (Its $z$ integral over the region between the plates diverges cubically.) As we have seen, this badly behaved function does not contribute to the force on the plates.

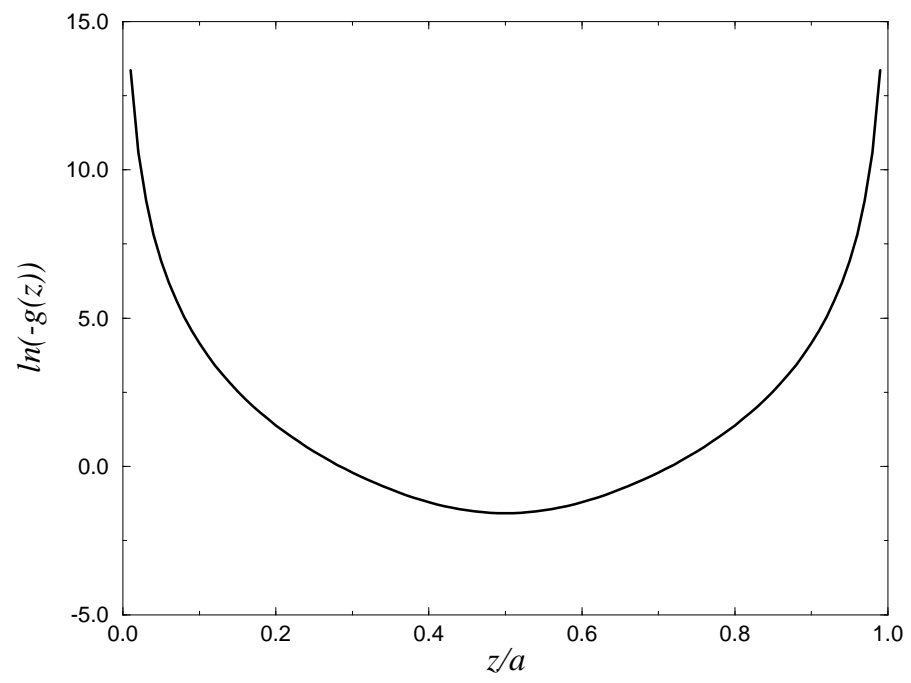

Figure 2. Local Casimir energy density between parallel plates.

Next, we turn to $\left\langle T_{z z}\right\rangle$. According to the stress tensor (10) and the Green's function (29), that is given by

$$
\begin{aligned}
& \left\langle T_{z z}\right\rangle=\frac{1}{2 i}\left(\partial_{z} \partial_{z}^{\prime}-\partial_{x} \partial_{x}^{\prime}-\partial_{y} \partial_{y}^{\prime}+\partial_{0} \partial_{0}^{\prime}\right) G\left(x, x^{\prime}\right) \\
= & -\frac{1}{2 i} \int \frac{d \omega d^{2} k}{(2 \pi)^{3}}\left(\partial_{z} \partial_{z}^{\prime}+\lambda^{2}\right) \frac{1}{\lambda \sin \lambda a} \sin \lambda z_{<} \sin \lambda\left(z_{>}-a\right) \\
= & -\frac{1}{2 i} \int \frac{d \omega d^{2} k}{(2 \pi)^{3}} \frac{\lambda}{\sin \lambda a}[\cos \lambda z \cos \lambda(z-a)+\sin \lambda z \sin \lambda(z-a)] \\
= & \int \frac{d \omega d^{2} k}{(2 \pi)^{3}} \frac{i \lambda}{2} \cot \lambda a,
\end{aligned}
$$


which is independent of $z$; that is, the normal-normal component of the expectation value of the stress tensor between the plates is constant. If once again, the irrelevant $a$-independent part is removed, what is left is just three times the constant part of the energy density (37),

$$
\left\langle T_{z z}\right\rangle=-3 \times \frac{\pi^{2}}{1440 a^{4}} .
$$

The remaining nonzero components of the stress tensor are

$$
\begin{aligned}
\left\langle T_{x x}\right\rangle & =\left\langle T_{y y}\right\rangle=\frac{1}{2 i}\left[\partial_{x} \partial_{x}^{\prime}-\partial_{y} \partial_{y}^{\prime}-\partial_{z} \partial_{z}^{\prime}+\partial_{0} \partial_{0}^{\prime}\right] G\left(x, x^{\prime}\right) \\
& =-\frac{1}{2 i} \int \frac{d \omega d^{2} k}{(2 \pi)^{3}} \frac{1}{\lambda \sin \lambda a}\left[\omega^{2} \sin \lambda z \sin \lambda(z-a)-\lambda^{2} \cos \lambda z \cos \lambda(z-a)\right] \\
& =-u-g(z),
\end{aligned}
$$

where we have again introduced polar coordinates in the frequency-wavenumber plane, and again dropped the infinite ( $a$-independent) constant in $u$. Thus the tensor structure of stress tensor is

$$
\left\langle T^{\mu \nu}\right\rangle(z)=u\left(\begin{array}{cccc}
1 & 0 & 0 & 0 \\
0 & -1 & 0 & 0 \\
0 & 0 & -1 & 0 \\
0 & 0 & 0 & 3
\end{array}\right)+g(z)\left(\begin{array}{cccc}
1 & 0 & 0 & 0 \\
0 & -1 & 0 & 0 \\
0 & 0 & -1 & 0 \\
0 & 0 & 0 & 0
\end{array}\right),
$$

where $u$ is given by (37) and $g$ by (40). Because $u$ is constant, this vacuum expectation value is divergenceless, since $g(z)$ does not contribute to $\left\langle T^{z z}\right\rangle$ :

$$
\partial_{\mu}\left\langle T^{\mu \nu}\right\rangle=\partial_{z}\left\langle T^{z z}\right\rangle=0
$$

The second term in (44) diverges at the boundaries, $z=0, a$, and has a integral over the volume which diverges; yet as we have seen, it is physically irrelevant because its integral is independent of $a$, and it has no normal component. Is there a natural way in which it simply does not appear in the local formulation?

The affirmative answer hinges on the ambiguity in defining the stress tensor. This ambiguity is without effect as far as the total stress or the total energy was concerned. Now, however, we see the virtue of the conformal stress tensol 20 .

$$
\tilde{T}^{\mu \nu}=\partial^{\mu} \phi \partial^{\nu} \phi-\frac{1}{2} g^{\mu \nu} \partial_{\lambda} \phi \partial^{\lambda} \phi-\frac{1}{6}\left(\partial^{\mu} \partial^{\nu}-g^{\mu \nu} \partial^{2}\right) \phi^{2},
$$

which, because of the equation of motion $\partial^{2} \phi=0$, has a vanishing trace,

$$
\tilde{T}_{\mu}^{\mu}=0 .
$$

If we use this stress tensor rather than the canonical one, we merely need supplement the above computations by that of the vacuum expectation value of the extra term. Thus to obtain $\left\langle\tilde{T}^{x x}\right\rangle$ we add to (43)

$$
\begin{aligned}
\frac{1}{6 i}\left(\partial_{y}^{2}+\partial_{z}^{2}-\partial_{0}^{2}\right) G(x, x) & =\frac{1}{6 i} \int \frac{d \omega d^{2} k}{(2 \pi)^{3}} \partial_{z}^{2}\left[-\frac{1}{\lambda \sin \lambda a} \sin \lambda z \sin \lambda(z-a)\right] \\
& =-\frac{1}{6 i} \int \frac{d \omega d^{2} k}{(2 \pi)^{3}} \frac{2 \lambda}{\sin \lambda z} \cos \lambda(2 z-a)=g(z),
\end{aligned}
$$


which just cancels the extra term in (43). Again, because $G(x, x)$ only depends on $z$, there is no extra contribution to $\left\langle T_{z z}\right\rangle$ :

$$
-\frac{1}{6}\left(\partial_{z}^{2}-g_{z z} \partial^{2}\right)\left\langle\phi^{2}\right\rangle=\frac{1}{6 i}\left(\partial_{x}^{2}+\partial_{y}^{2}-\partial_{0}^{2}\right) G(x, x)=0 .
$$

The extra term for $\left\langle T_{00}\right\rangle$ is just the negative of that in (48),

$$
-\frac{1}{6 i} \partial_{z}^{2} G(x, x)=-g(z),
$$

which cancels the second term in (36). Thus, the conformal stress tensor has the following vacuum expectation value for the region between the parallel plates:

$$
\left\langle\tilde{T}^{\mu \nu}\right\rangle=u\left(\begin{array}{cccc}
1 & 0 & 0 & 0 \\
0 & -1 & 0 & 0 \\
0 & 0 & -1 & 0 \\
0 & 0 & 0 & 3
\end{array}\right)
$$

which is traceless, thereby respecting the conformal invariance of the massless theory. This is just the result found by Brown and Maclay by general considerations 10 who argued that

$$
\left\langle\tilde{T}^{\mu \nu}\right\rangle=u\left[4 \hat{z}^{\mu} \hat{z}^{\nu}-g^{\mu \nu}\right],
$$

where $\hat{z}^{\mu}$ is the unit vector in the $z$ direction.

\subsection{Massive Scalar in D Spatial Dimensions}

It is instructive to repeat the above calculation for a massive scalar field where the plates have $D-1$ transverse dimensions. We will use the conformal stress tensor,

$$
T^{\mu \nu}=\partial^{\mu} \phi \partial^{\nu} \phi-\frac{1}{2} g^{\mu \nu}\left(\partial_{\lambda} \phi \partial^{\lambda} \phi+\mu^{2} \phi^{2}\right)-\alpha\left(\partial^{\mu} \partial^{\nu}-g^{\mu \nu} \partial^{2}\right) \phi^{2} .
$$

Here $\alpha$ has to be chosen to be $(D-1) /(4 D)$ in order that the trace vanish (by virtue of the field equations) in the massless limit:

$$
\alpha=\frac{D-1}{4 D}: \quad T_{\mu}^{\mu}=-\mu^{2} \phi^{2} .
$$

The calculation proceeds very similarly to that given above. The only new element is writing the momentum integral in polar coordinates:

$$
d^{D-1} k=\frac{2 \pi^{(D-1) / 2}}{\Gamma\left(\frac{D-1}{2}\right)} k^{D-2} d k
$$

and then introducing polar coordinates as in Eq. (32). We encounter the integrals

$$
\int_{0}^{\pi / 2} d \theta(\sin \theta)^{D-2}=2^{D-3} \frac{\Gamma\left(\frac{D-1}{2}\right)^{2}}{\Gamma(D-1)}
$$

relative to which

$$
\left\langle\sin ^{2} \theta\right\rangle=\frac{D-1}{D}, \quad\left\langle\cos ^{2} \theta\right\rangle=\frac{1}{D} .
$$


The result for the various nonzero components of the stress tensor are $\left(\kappa^{2}=\rho^{2}+\mu^{2}\right)$

$$
\begin{aligned}
& \left\langle T^{00}\right\rangle=-\frac{2^{-D} \pi^{-D / 2}}{D \Gamma(D / 2)} \int_{0}^{\infty} d \rho \rho^{D-1} \frac{1}{\kappa \sinh \kappa a}\left[\rho^{2} \cosh \kappa a+\mu^{2} \cosh \kappa(2 z-a)\right], \\
& \left\langle T^{z z}\right\rangle=-\frac{2^{-D} \pi^{-D / 2}}{\Gamma(D / 2)} \int_{0}^{\infty} d \rho \rho^{D-1} \kappa \operatorname{coth} \kappa a, \\
& \left\langle T^{x x}\right\rangle=\left\langle T^{y y}\right\rangle=\cdots=-\left\langle T^{00}\right\rangle .
\end{aligned}
$$

Surface divergent terms, which do not contribute to the observable force, appear proportional to the square of the mass. Now, the trace of the expectation value of the stress tensor is nonzero because of the mass:

$$
\begin{aligned}
\left\langle T_{\mu}^{\mu}\right\rangle & =\left\langle T^{z z}\right\rangle-D\left\langle T^{00}\right\rangle=-\mu^{2}\left\langle\phi^{2}\right\rangle \\
& =-\mu^{2} \frac{2^{-D} \pi^{-D / 2}}{\Gamma(D / 2)} \int_{0}^{\infty} d \rho \rho^{D-1} \frac{1}{\kappa \sinh \kappa a}[\cosh \kappa a-\cosh \kappa(2 z-a)] .
\end{aligned}
$$

Of course, the infinite $a$-independent stress which would be present if the boundary were not present $(\operatorname{coth} \kappa a \rightarrow 1)$ is to be removed. The well known 21 expressions for the force and the energy between parallel plates may be easily recovered. We do not see the necessity for the additional terms found by Graham et al 13 in the energy to make the energy finite at zero separation (the fact that the Casimir energy diverges at $a=0$ reflects the infinite amount of energy released when the plates are pushed into coincidence) nor the requirement that the energy should be infinite at zero mass, when the observable force is finite there.

\section{Scalar Casimir Effect for a Dirichlet Sphere}

The calculation given in the first part of this talk was that for a sphere in one spatial dimension. Now we consider a massless scalar in three space dimensions, with a spherical boundary on which the field vanishes. This corresponds to the TE modes for the electrodynamic situation first solved by Boyer 4 The general calculation in $D$ dimensions was given in Bender and Milton 7 , the force per unit area is given by the formula

$$
\mathcal{F}=-\sum_{l=0}^{\infty} \frac{(2 l+D-2) \Gamma(l+D-2)}{l ! 2^{D} \pi^{(D+1) / 2} \Gamma\left(\frac{D-1}{2}\right) a^{D+1}} \int_{0}^{\infty} d x x \frac{d}{d x} \ln \left[I_{\nu}(x) K_{\nu}(x) x^{2-D}\right] .
$$

Here $\nu=l-1+D / 2$. For $D=3$ this expression reduces to

$$
\mathcal{F}=-\frac{1}{8 \pi^{2} a^{4}} \sum_{l=0}^{\infty}(2 l+1) \int_{0}^{\infty} d x x \frac{d}{d x} \ln \left[I_{l+1 / 2}(x) K_{l+1 / 2}(x) / x\right] .
$$

In Ref. 7 we evaluated this expression by continuing in $D$ from a region where both the sum and integrals existed. In that way, a completely finite result was found for all positive $D$ not equal to an even integer. Here we will adopt a perhaps more physical approach, that of allowing the time coordinates in the underlying Green's function to approach each other, as described in Ref. 22 That is, we recognize 
that the $x$ integration above is actually a (dimensionless) frequency integral, and therefore we should replace

$$
\int_{0}^{\infty} d x f(x)=\frac{1}{2} \int_{-\infty}^{\infty} d y e^{i y \delta} f(|y|)
$$

where at the end we are to take $\delta \rightarrow 0$. Immediately, we can replace the $x^{-1}$ inside the logarithm in Eq. (63) by $x$, which makes the integrals converge, because the difference is proportional to a delta function in the time separation, a contact term without physical significance.

To proceed, we use the uniform asymptotic expansions for the modified Bessel functions. This is an expansion in inverse powers of $\nu=l+1 / 2$, low terms in which turn out to be remarkably accurate even for modest $l$. The leading terms in this expansion are

$$
\ln \left[x I_{l+1 / 2}(x) K_{l+1 / 2}(x)\right] \sim \ln \frac{z t}{2}+\frac{1}{\nu^{2}} g(t)+\frac{1}{\nu^{4}} h(t)+\ldots,
$$

where $x=\nu z$ and $t=\left(1+z^{2}\right)^{-1 / 2}$. Here

$$
\begin{aligned}
& g(t)=\frac{1}{8}\left(t^{2}-6 t^{4}+5 t^{6}\right), \\
& h(t)=\frac{1}{64}\left(13 t^{4}-284 t^{6}+1062 t^{8}-1356 t^{10}+565 t^{12}\right) .
\end{aligned}
$$

The leading term in the force/area is therefore

$$
\mathcal{F}_{0}=-\frac{1}{8 \pi^{2} a^{4}} \sum_{l=0}^{\infty}(2 l+1) \nu \int_{0}^{\infty} d z t^{2}=-\frac{1}{8 \pi a^{4}} \sum_{l=0}^{\infty} \nu^{2}=\frac{3}{32 \pi a^{4}} \zeta(-2)=0,
$$

where in the last step we have used a formal zeta function evaluation. ${ }^{a}$ Here the rigorous way to argue is to recall the presence of the point-splitting factor $e^{i \nu z \delta}$ and to carry out the sum on $l$ using

$$
\sum_{l=0}^{\infty} e^{i \nu z \delta}=-\frac{1}{2 i} \frac{1}{\sin z \delta / 2}
$$

so

$$
\sum_{l=0}^{\infty} \nu^{2} e^{i \nu z \delta}=-\frac{d^{2}}{d(z \delta)^{2}} \frac{i}{2 \sin z \delta / 2}=\frac{i}{8}\left(-\frac{2}{\sin ^{3} z \delta / 2}+\frac{1}{\sin z \delta / 2}\right) .
$$

Then $\mathcal{F}_{0}$ is given by the divergent expression

$$
\mathcal{F}_{0}=\frac{i}{\pi^{2} a^{4} \delta^{3}} \int_{-\infty}^{\infty} \frac{d z}{z^{3}} \frac{1}{1+z^{2}}
$$

which we argue is zero because the integrand is odd.

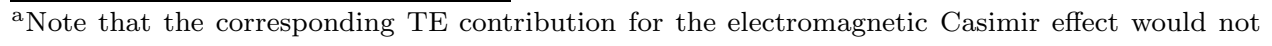
be zero, for there the sum starts from $l=1$. 
The next term in the uniform asymptotic expansion (65), that involving $g$, is likewise zero, as intimated by the formal zeta function identity,

$$
\sum_{l=0}^{\infty} \nu^{s}=\left(2^{-s}-1\right) \zeta(-s)
$$

which vanishes at $s=0$. The same conclusion follows from point splitting, as we can see through use of the Euler-Maclaurin sum formula,

$$
\sum_{l=0}^{\infty} f(l)=\int_{0}^{\infty} d l f(l)+\frac{1}{2} f(0)-\sum_{k=1}^{\infty} \frac{B_{k}}{(2 k) !} f^{(2 k-1)}(0) .
$$

Here we have

$$
\int_{0}^{\infty} d l e^{i \nu z \delta}=-\frac{e^{i z \delta / 2}}{i z \delta}=-\frac{1}{i z \delta}-\frac{1}{2}+\mathcal{O}(\delta) .
$$

We argue again that the first term here gives no contribution to the integral over $z$ because it is odd, and then the first two terms in the Euler-Maclaurin formula give

$$
\mathcal{F}_{1}=-\frac{1}{8 \pi^{2} a^{4}}\left[-\frac{1}{2} \int_{-\infty}^{\infty} d z z \frac{d}{d z} g(t)+\frac{1}{2} \int_{-\infty}^{\infty} d z z \frac{d}{d z} g(t)\right]=0 .
$$

Derivatives of $e^{i \nu z \delta}$ with respect to $l$ all vanish at $\delta=0$. Again, this cancellation does not occur in the electromagnetic case because there the sum starts at $l=1$. So here the leading term which survives is that of order $\nu^{-4}$ in Eq. 655), namely

$$
\mathcal{F}_{1}=\frac{1}{4 \pi^{2} a^{4}} \sum_{l=0}^{\infty} \frac{1}{\nu^{2}} \int_{0}^{\infty} d z h(t),
$$

where we have now dropped the point splitting factor because this expression is completely convergent. The integral over $z$ is

$$
\int_{0}^{\infty} d z h(t)=\frac{35 \pi}{32768}
$$

and the sum over $l$ is $3 \zeta(2)=\pi^{2} / 2$, so the leading contribution to the stress on the sphere is

$$
\mathcal{S}_{2}=4 \pi a^{2} \mathcal{F}_{2}=\frac{35 \pi^{2}}{65536 a^{2}}=\frac{0.00527094}{a^{2}} .
$$

Numerically this is a terrible approximation.

What we must do now is return to the full expression and add and subtract the leading asymptotic terms. This gives

$$
\mathcal{S}=\mathcal{S}_{2}-\frac{1}{2 \pi a^{2}} \sum_{l=0}^{\infty}(2 l+1) R_{l},
$$

where

$$
R_{l}=Q_{l}+\int_{0}^{\infty} d x\left[\ln z t+\frac{1}{\nu^{2}} g(t)+\frac{1}{\nu^{4}} h(t)\right]
$$


where the integral

$$
Q_{l}=-\int_{0}^{\infty} d x \ln \left[2 x I_{\nu}(x) K_{\nu}(x)\right]
$$

has the asymptotic form $(l \gg 1)$

$$
Q_{l} \sim \frac{\nu \pi}{2}+\frac{\pi}{128 \nu}-\frac{35 \pi}{32768 \nu^{3}}+\frac{565 \pi}{1048577 \nu^{5}}-\frac{1208767 \pi}{2147483648 \nu^{7}}+\frac{138008357 \pi}{137438953472 \nu^{9}} .
$$

The first two terms in Eq. (81) cancel the second and third terms in Eq. (79), of course. The third term in Eq. (81) corresponds to $h(t)$, so the last three terms displayed in Eq. (81) give the asymptotic behavior of the remainder, which we call $w(\nu)$. Then we have, approximately,

$$
\mathcal{S} \approx \mathcal{S}_{2}-\frac{1}{\pi a^{2}} \sum_{l=0}^{n} \nu R_{l}-\frac{1}{\pi a^{2}} \sum_{l=n+1}^{\infty} \nu w(\nu) .
$$

For $n=1$ this gives $\mathcal{S} \approx 0.00285278 / a^{2}$, and for larger $n$ this rapidly approaches the value first given in Bender and Milton 7 :

$$
\mathcal{S}=0.002817 / a^{2}
$$

a value much smaller than the famous electromagnetic result 4 [22

$$
\mathcal{S}^{\mathrm{EM}}=\frac{0.04618}{a^{2}},
$$

because of the cancellation of the leading terms noted above.

\section{Diagrammatic Divergence Structure}

So far we have come to rather different conclusions from those of Graham et al.13 For the case of parallel plates, we found:

- The massless theory is perfectly well defined (no infrared divergences), and surface divergences, which in any case have no physical consequences, do not appear if the conformal stress tensor is used.

- The vacuum expectation value of the stress tensor for the case of a massive scalar does have surface divergences, which are proportional to the mass squared, but which do not contribute to the force and are therefore physically irrelevant.

For a massless scalar with a spherical boundary in three dimensions, the formal expressions for the force/area and the energy are formally divergent, yet if they are regulated, say by point-splitting, the divergences cancel and the energy and self-stress on the sphere are completely finite and unambiguous.

Graham et al 13 dispute this. However, their disagreement with us on the $D=1$ case seems entirely semantic, and without observable consequence. Their 
substantial argument hinged on their $D=2$ calculation. However, it is well known that the Casimir effect for a circle is divergent, so it is hard to draw general inferences from an examination of that situation. ${ }^{\mathrm{b}}$ Here, we will re-examine some of their general arguments for a hypersphere in $D$ space dimensions.

The general analysis for that case was given in Bender and Milton 7 ; it is clear that the point-splitting method given in the previous section could be applied in that analysis. Instead, we will here focus on the issue of the second-order Feynman graph which supposedly is the signal for the divergence of the theory in any number of space dimensions. (It is the oversubtracted graph which leaves the mode sum more convergent.) We will adopt a somewhat simpler formalism than that given by Graham et al. 13 based on the "trace-log" formula for the energy,

$$
E=\frac{i}{2 T} \operatorname{Tr} \ln G
$$

where for a "polarization" operator $\Pi$

$$
G=G_{0}(1+\Pi G)=G_{0}\left(1+\Pi G_{0}+\Pi G_{0} \Pi G_{0}+\ldots\right) .
$$

The highly sensible approach of Graham et al 13 is to replace ideal boundary conditions by an interaction with an external field $\sigma$. The Lagrangian for the scalar field is thus taken to be

$$
\mathcal{L}=-\frac{1}{2}\left(\partial_{\mu} \phi \partial^{\mu} \phi+m^{2} \phi^{2}+\sigma(r) \phi^{2}\right),
$$

where, anticipating spherical symmetry, we have taken the external field to depend only on the spatial radial coordinate. In the end, we may take $\sigma$ to be a delta function,

$$
\sigma(r)=\frac{g}{a} \delta(r-a)
$$

where $g$ is dimensionless and the formal $g \rightarrow \infty$ limit corresponds to the situation of a Dirichlet spherical shell. We can now evaluate the one-loop vacuum energy by the replacement $\Pi \rightarrow \sigma$ in Eqs. 855), 866). It is the second-order graph that is supposed to signal nonrenormalizability.

We carry out the calculation in $D$ dimensions. ${ }^{\mathrm{c}}$

$$
\begin{aligned}
E & =\frac{1}{2} \frac{i}{2 T} \operatorname{Tr} \sigma G_{0} \sigma G_{0} \\
& =\frac{i}{4 T} \int d^{D+1} x d^{D+1} y \sigma(x) G_{0}(x-y) \sigma(y) G_{0}(y-x) \\
& =\frac{i}{4} \int d^{D} x d^{D} y \sigma(|x|) \sigma(|y|) \int \frac{d \omega}{2 \pi} \int \frac{d^{D} p}{(2 \pi)^{D}} \frac{d^{D} q}{(2 \pi)^{D}} \frac{e^{i(\mathbf{p}-\mathbf{q}) \cdot(\mathbf{x}-\mathbf{y})}}{\left(p^{2}+m^{2}\right)\left(q^{2}+m^{2}\right)}
\end{aligned}
$$

\footnotetext{
${ }^{\mathrm{b}}$ However, in their most recent papers, Graham et al 14 consider a three-dimensional $\delta$-shell potential, and show a divergence occurs there not only in second order, which we do not find, but in third order, with which we concur 16

${ }^{\mathrm{c}}$ Equation [89] incorporates a net $1 /(4 \pi)$ correction relative to the formula (4.4) in Ref. 17
} 
where in the last line we have carried out the integral on $t$ and $t^{\prime}$, and as a result $p^{0}=q^{0}=\omega$. Now we introduce polar coordinates, so in terms of the last angle

$$
d^{D} x=A_{D-1} x^{D-1} d x \sin ^{D-2} \theta d \theta,
$$

where $A_{n}=2 \pi^{n / 2} / \Gamma(n / 2)$ is the surface area of a sphere in $n$ dimensions. Then we encounter a Bessel function

$$
\int_{0}^{\pi} d \theta \sin ^{D-2} \theta e^{i|\mathbf{p}-\mathbf{q}| x \cos \theta}=\left(\frac{2}{|\mathbf{p}-\mathbf{q}| x}\right)^{D / 2-1} \sqrt{\pi} \Gamma\left(\frac{D-1}{2}\right) J_{D / 2-1}(|\mathbf{p}-\mathbf{q}| x) .
$$

Thus the Fourier transform of the field $\sigma(|x|)$ is defined by

$$
\tilde{\sigma}(k)=\int d^{D} x e^{i \mathbf{k} \cdot \mathbf{x}} \sigma(x)=k\left(\frac{2 \pi}{k}\right)^{D / 2} \int_{0}^{\infty} d x x^{D / 2} J_{D / 2-1}(k x) \sigma(x) .
$$

(This agrees with the expression in Graham et al. for $D=2$.) The expression for the energy reduces to

$$
E=\left.\frac{i}{4} \int \frac{d \omega}{2 \pi} \int \frac{d^{D} q d^{D} p}{(2 \pi)^{2 D}} \frac{\tilde{\sigma}(|\mathbf{p}-\mathbf{q}|)^{2}}{\left(p^{2}+m^{2}\right)\left(q^{2}+m^{2}\right)}\right|_{p^{0}=q^{0}=\omega} .
$$

We carry out the momentum integrations by first using the proper-time representation to combine the denominators:

$$
\begin{aligned}
\frac{1}{p^{2}+m^{2}} \frac{1}{q^{2}+m^{2}} & =-\int_{0}^{\infty} d s \int_{0}^{\infty} d s^{\prime} e^{-i s\left(p^{2}+m^{2}\right)-i s^{\prime}\left(q^{2}+m^{2}\right)} \\
& =-\int_{0}^{\infty} d s s \int_{0}^{1} d u e^{-i s m^{2}-i s(1-u) p^{2}-i s u q^{2}},
\end{aligned}
$$

where in the second line we replace $s \rightarrow s(1-u), s^{\prime} \rightarrow s u$. In terms of $\mathbf{k}=\mathbf{p}-\mathbf{q}$, we complete the square in the exponent by writing

$$
s(1-u) \mathbf{p}^{2}+s u \mathbf{q}^{2}=s\left[(\mathbf{p}-u \mathbf{k})^{2}+\mathbf{k}^{2} u(1-u)\right],
$$

while the corresponding 0 components combine to give $-s \omega^{2}$. Now the frequency and $\mathbf{p}$ integrals are just Gaussian:

$$
\int d \omega e^{i s \omega^{2}}=e^{i \pi / 4} \sqrt{\frac{\pi}{s}}, \quad \int d^{D}(p-u k) e^{-i s(\mathbf{p}-u \mathbf{k})^{2}}=e^{-i \pi D / 4}\left(\frac{\pi}{s}\right)^{D / 2} .
$$

Finally, we introduce polar coordinates for the $\mathbf{k}$ integration, with the result

$$
E=-\frac{\pi^{-D-1 / 2}}{2^{2+2 D}} \frac{\Gamma\left(\frac{3-D}{2}\right)}{\Gamma\left(\frac{D}{2}\right)} \int_{0}^{\infty} d k k^{D-1} \tilde{\sigma}(k)^{2} \int_{0}^{1} d u\left[m^{2}+u(1-u) k^{2}\right]^{D / 2-3 / 2},
$$

which yields the $D=2$ result given by Graham et al 13

If we choose a delta-function potential,

$$
\sigma(x)=\frac{g}{a} \delta(x-a)
$$


we obtain

$$
E=-\frac{\pi^{-1 / 2}}{2^{2+D}} \frac{\Gamma\left(\frac{3-D}{2}\right)}{\Gamma\left(\frac{D}{2}\right)} \frac{g^{2}}{a} \int_{0}^{\infty} d \xi \xi J_{D / 2-1}^{2}(\xi) \int_{0}^{1} d u\left[m^{2} a^{2}+\xi^{2} u(1-u)\right]^{(D-3) / 2}
$$

This appears to converge for $0<D<2$ except for the exceptional case $m=0$. In that case the $u$ integral is simply

$$
\frac{\Gamma\left(\frac{D-1}{2}\right)^{2}}{\Gamma(D-1)}=2^{2-D} \pi^{1 / 2} \frac{\Gamma\left(\frac{D-1}{2}\right)}{\Gamma\left(\frac{D}{2}\right)}
$$

and the integral over the Bessel functions is

$$
\begin{aligned}
\int_{0}^{\infty} d \xi \xi^{D-2} J_{D / 2-1}^{2}(\xi) & =2^{D-2} \frac{\Gamma(2-D) \Gamma(D-3 / 2)}{\Gamma\left(\frac{3-D}{2}\right)^{2} \Gamma\left(\frac{1}{2}\right)} \\
& =\frac{\Gamma(1-D / 2) \Gamma(D-3 / 2)}{2 \pi \Gamma\left(\frac{3-D}{2}\right)},
\end{aligned}
$$

which is valid in the region $\frac{3}{2}<D<2$. Thus the energy for a massless scalar is

$$
E=-2^{-1-2 D} \frac{g^{2}}{\pi a} \frac{\Gamma\left(\frac{D-1}{2}\right) \Gamma(D-3 / 2) \Gamma(1-D / 2)}{\Gamma\left(\frac{D}{2}\right)^{2}},
$$

which we take to be the appropriate analytic continuation for all $D$. This exhibits poles at $D=2,4,6, \ldots$, in congruence with the known divergence structure of the Casimir effect. There are also poles occurring at $D=1,-1,-3, \ldots$, and at $D=$ $3 / 2,1 / 2,-1 / 2, \ldots$ These latter two sequences of divergent dimensions correspond to infrared divergences that have no counterpart in the Casimir calculations, unlike the ultraviolet, even-integer poles. For space dimension between 2 and 4 the Casimir energy is completely finite, in concert with this diagnostic. The divergence at $D=2$, even putting aside the question of mass, is seen not to be generic.

Instead of dimensional continuation, one can work directly in $D=3$. Let us regulate the theory by inserting a lower limit $s_{0} \rightarrow 0$ in the proper-time integration, so that for $m=0$ the energy (97) becomes

$$
\begin{aligned}
E & =\frac{1}{2^{7} \pi^{4}} \int_{0}^{\infty} d k k^{2} \tilde{\sigma}(k)^{2} \int_{0}^{1} d u \ln \left[s_{0} k^{2} u(1-u)\right] \\
& =\left.\frac{1}{2^{7} \pi^{4}} \int_{0}^{\infty} d k k^{2} \tilde{\sigma}(k)^{2} \frac{d}{d \alpha} \int_{0}^{1} d u\left[s_{0} k^{2} u(1-u)\right]^{\alpha}\right|_{\alpha=0} .
\end{aligned}
$$

If the derivative acts on anything but $k^{2 \alpha}$ we have

$$
\int_{0}^{\infty} d k k^{2} \tilde{\sigma}(k)^{2}=(2 \pi)^{3} \int_{0}^{\infty} d x x^{2} \sigma(x)^{2} .
$$

This diverges as $\sigma(x) \rightarrow(g / a) \delta(x-a)$; but if we regulate the divergence by pointsplitting

$$
\sigma(x)^{2} \rightarrow \lim _{\xi \rightarrow \infty} \sigma(x-\xi) \sigma(x+\xi)
$$


we have

$$
\int_{0}^{\infty} d k k^{2} \tilde{\sigma}(k)^{2}=(2 \pi)^{3} g^{2} \delta(2 \xi)
$$

which is seen to be a contact term, independent of $a$. We are left with

$$
E=\left.\frac{1}{8 \pi^{2}} \int_{0}^{\infty} d x x \sigma(x) \int_{0}^{\infty} d y y \sigma(y) \frac{d}{d \alpha} \int_{0}^{\infty} d k k^{2 \alpha} \sin k x \sin k y\right|_{\alpha=0} .
$$

Here we have used the Fourier transformation expression (92), but replaced Bessel functions of order $1 / 2$ by the corresponding trigonometric functions. The $k$ integral is now obtained from $(-1<\alpha<0)$

$$
\int_{0}^{\infty} d x x^{\alpha} \cos \beta x=\frac{\Gamma(\alpha+1) \cos (\alpha+1) \frac{\pi}{2}}{\beta^{\alpha+1}}
$$

which gives for the energy

$$
E=\frac{1}{16 \pi} \int_{0}^{\infty} d x x \sigma(x) \int_{0}^{\infty} d y y \sigma(y)\left(\frac{1}{x+y}-\frac{1}{|x-y|}\right) \rightarrow \frac{g^{2}}{32 \pi a},
$$

where we have omitted another infinite term that is independent of $a$. The result is exactly the $D=3$ value of Eq. (102)! The justification for omitting (infinite) constant terms in the energy is that they are unobservable, not corresponding to a self-stress on the sphere.

\subsection{Third-Order Divergence}

In their most recent papers, Graham et al ${ }^{14}$ explicitly consider a three-dimensional spherical shell, and show that with a $\delta$-function potential a divergence occurs not only in second order (which they acknowledge might be redefined away) but also in third order. The former is, as we have seen, illusory, while the latter is a real, logarithmic divergence, first discovered some years ago by Bordag et al 15 I have recently carried out a complete reconsideration of the Casimir self-stress due $\delta$ function potentials, reproduced the $\mathcal{O}\left(g^{2}\right)$ energy in Eq. (108), and identified the $\mathcal{O}\left(g^{3}\right)$ divergence for the spherical shell configuration. It is possible that this divergence could be cancelled in the electromagnetic case between TE and TM modes. For further details see Ref. 16.

\section{Conclusions}

The challenge set forth by Graham et al 1314 is physically appropriate and timely given the development of our understanding of the Casimir effect. Certainly those authors are justified in objecting to the loose use of the term "renormalization" in connection with various dubious processes for removing divergences in boundaryvalue Casimir problems. However, it is important to separate the wheat from the chaff. The Casimir force between parallel plates, the self-stress (or the force per unit area) on a perfect (Dirichlet or Neumann) spherical or cylindrical shell due to 
massless fields, the energy of fields confined to a curved manifold (a hypersphere or torus for example) are examples where the Casimir energy is unambiguous and finite, except for exceptional numbers of spatial dimensions. Of course, these are special cases, and generically Casimir energies are infinite. This is true if fields bounded by a spherical shell have mass, if the shell has finite thickness, or if the speed of light inside and outside the shell has different values. The latter case is the interesting one of a dielectric ball. The stress or the energy in that case is quartically divergent. I argued, very tentatively in 1980, 11 and others argued more forcefully later, 12 that the divergent terms could be reabsorbed into the definition of physical properties of the material medium, the mass density, surface tension, and the like. This "renormalization" was in the spirit of the first use of renormalization in physics. 24 Obviously, this was not an altogether convincing argument, but it was argued to be on a par with perturbative renormalization of a quantum field theory.25. However, fairly recently, the discovery by several groups 26 that the finite part of the Casimir energy for a dilute dielectric sphere was unique, and coincided with that obtained by a regulated (dimensionally continued) calculation of the van der Waals energy, 27 did provide some evidence that the divergences could be removed unambiguously, ${ }^{\mathrm{d}}$ and had the practical consequence of destroying the hope of explaining sonoluminescence on the basis of quantum vacuum energy?28

Obviously we are still at the early stages of understanding quantum field theory. The nature of divergences in vacuum energy calculations is still not understood. However, there are a few established peaks that rise above the murky clouds of ignorance, and we should not abandon them lightly because the rest is obscure.

\section{Acknowledgments}

I am grateful to the US Department of Energy for partial support of this research. I thank Bob Jaffe, Steve Fulling, and Michael Bordag for helpful conversations. I thank Vladimir Mostepanenko for inviting me to participate in MGX.

\section{References}

1. H. B. G. Casimir and D. Polder, Phys. Rev. 73, 360 (1948).

2. H. B. G. Casimir, J. Chim. Phys. 46, 407 (1949).

3. H. B. G. Casimir, Proc. Kon. Ned. Akad. Wetensch. 51, 793 (1948).

4. T. H. Boyer, Phys. Rev. 174, 1764 (1968).

5. L. L. DeRaad, Jr., and K. A. Milton, Ann. Phys. (N.Y.) 136, 229 (1981).

6. S. Sen, Phys. Rev. D 24, 869 (1981); J. Math. Phys. 22, 869 (1981).

7. C. M. Bender and K. A. Milton, Phys. Rev. D 50, 6547 (1994).

8. K. A. Milton, Phys. Rev. D 55, 4940 (1997).

9. D. Deutsch and P. Candelas, Phys. Rev. D 20, 3063 (1979).

10. L. S. Brown and G. J. Maclay, Phys. Rev. 184, 1272 (1969).

d This divergence of the Casimir energy in order $(\epsilon-1)^{3}$ for a dilute dielectric ball is analogous to the divergence of the Casimir energy of a $\delta$-function sphere at $\mathcal{O}\left(g^{3}\right)$, as noted in Ref. 15] 
11. K. A. Milton, Ann. Phys. (N.Y.) 127, 49 (1980).

12. M. Bordag, E. Elizalde, K. Kirsten, and S. Leseduarte, Phys. Rev. D 56, 4896 (1997); M. Scandurra, J. Phys. A 33, 5707 (2000); S. K. Blau, M. Visser, and A. Wipf, Nucl. Phys. B 310. 163 (1988).

13. N. Graham, R. L. Jaffe, M. Quandt, and H. Weigel, Phys. Rev. Lett. 87, 131601 (2001); N. Graham, R. L. Jaffe, and H. Weigel, Int. J. Mod. Phys. A 17, 846 (2002); N. Graham, R. L. Jaffe, V. Khemani, M. Quandt, M. Scandurra, and H. Weigel, Nucl. Phys. B 645, 49 (2002); Phys. Lett. B 572, 196 (2003).

14. N. Graham, R. L. Jaffe, V. Khemani, M. Quandt, O. Schroeder, and H. Weigel, Nucl. Phys. B677, 379 (2004); H. Weigel, hep-th/0310301

15. M. Bordag, K. Kirsten, and D. Vassilevich, Phys. Rev. D 59, 085011 (1999).

16. K. A. Milton, hep-th/0401090

17. K. A. Milton, Phys. Rev. D 68, 065020 (2003).

18. M. Lüscher, K. Symanzik, and P. Weisz, Nucl. Phys. B 173, 365 (1980).

19. K. A. Milton, The Casimir Effect: Physical Manifestations of Zero-Point Energy (World Scientific, Singapore, 2001).

20. C. G. Callan, Jr., S. Coleman, and R. Jackiw, Ann. Phys. (N.Y.) 59, 42 (1970).

21. J. Ambjørn and S. Wolfram, Ann. Phys. (N.Y.) 147, 1 (1983).

22. K. A. Milton, L. L. DeRaad, Jr., and J. Schwinger, Ann. Phys. (N.Y.) 115, 388 (1978).

23. M. Bordag, E. Elizalde, K. Kirsten, and S. Leseduarte, Phys. Rev. D 56, 4896 (1997).

24. S. D. Poisson, Mem. Acad. Sci. xl, 521 (1832), quoted in H. Lamb, Hydrodynamics (Dover, New York, 1945).

25. M. Bordag, A. S. Goldhaber, P. van Nieuwenhuizen, and D. Vassilevich, Phys. Rev. D 66125014 (2002).

26. I. Brevik, V. N. Marachevsky, and K. A. Milton, Phys. Rev. Lett. 82, 3948 (1999); G. Barton, J. Phys. A 32, 525 (1999); M. Bordag, K. Kirsten, and D. Vassilevich, Phys. Rev. D 59, 085011 (1999); J. S. Høye and I. Brevik, J. Stat. Phys. 100, 223 (2000); G. Lambiase, G. Scarpetta, and V. V. Nesterenko, Mod. Phys. Lett. A 16, 1893 (2001).

27. K. A. Milton and Y. J. Ng, Phys. Rev. E 55, 4207 (1997).

28. M. P. Brenner, S. Hilgenfeldt, and D. Lohse, Rev. Mod. Phys. 74, 425 (2002). 